\title{
FRAÇÕES DE NITROGÊNIO EM LUVISSOLO SOB SISTEMAS AGROFLORESTAIS E CONVENCIONAL NO SEMI-ÁRIDO CEARENSE ${ }^{(1)}$
}

\author{
Stoécio Malta Ferreira Maia ${ }^{(2)}$, Francisco Alisson da Silva Xavier ${ }^{(3)}$, \\ Teógenes Senna de Oliveira ${ }^{(4)}$, Eduardo de Sá Mendonça ${ }^{(5)}$ \& João \\ Ambrósio Araújo Filho ${ }^{(6)}$
}

\begin{abstract}
RESUMO
Os sistemas agroflorestais têm sido amplamente promovidos como sistemas de produção agrícola sustentáveis, principalmente para regiões subdesenvolvidas, onde o uso de insumos externos é inviável. Este trabalho objetivou avaliar o impacto de quatro sistemas agroflorestais e um sistema convencional sobre os teores de $\mathbf{N}$ total, mineral e em diferentes frações orgânicas, após cinco anos de uso de um Luvissolo na região semi-árida cearense, em experimento instalado na Embrapa Caprinos, em Sobral (CE). Os sistemas testados foram: agrossilvipastoril (AGP); silvipastoril (SILV); tradicional cultivado em 1998 e 1999 (TR98); tradicional cultivado em 2002 (TR02); cultivo convencional (CC); e uma área de Caatinga (CA). Nas amostras de solo, avaliaram-se os teores de $\mathrm{N}$ total, $\mathrm{N}-\mathrm{NH}_{4}{ }^{+}, \mathrm{N}-\mathrm{NO}_{3}{ }^{-}, \mathrm{N}$ microbiano, $\mathbf{N}$ da matéria orgânica leve (livre e oclusa) e o $\mathbf{N}$ das substâncias húmicas. Os resultados indicaram que todos os tratamentos condicionaram elevados teores de $\mathrm{N}-\mathrm{NO}_{3}{ }^{-}$, representando entre 10,3 e 23,5 \% dos teores de $\mathrm{N}$ total. $\mathrm{O}$ sistema $\mathrm{CC}$ reduziu os teores de $\mathbf{N}$ total e das frações das substâncias húmicas em 38 e $44 \%$, respectivamente, na camada superficial do solo. Dentre os sistemas agroflorestais, os sistemas AGP e TR98 causaram redução significativa dos teores de $\mathbf{N}$ total, $\mathbf{N}$ da matéria orgânica leve (livre e oclusa) e N das substâncias húmicas.
\end{abstract}

\footnotetext{
(1) Parte da Tese de Mestrado do primeiro autor apresentada ao programa de Pós-Graduação em Agronomia, Universidade Federal do Ceará - UFC. Recebido para publicação em abril de 2006 e aprovado em setembro de 2007.

${ }^{(2)}$ Doutorando em Solos e Nutrição de Plantas da Escola Superior de Agricultura "Luiz de Queiroz", Universidade de São Paulo - ESALQ/USP. Laboratório de Biogeoquímica Ambiental - CENA. Av. Centenário 303, CEP 13400-970 Piracicaba (SP). E-mail: smfmaia@esalq.usp.br

${ }^{(3)}$ Doutorando em Solos da Universidade Federal de Viçosa - UFV. Av. PH Rolfs sn, CEP 36750-000 Viçosa (MG). Email: ali_xavier@yahoo.com.br

${ }^{(4)}$ Professor do Departamento de Ciências do Solo, Universidade Federal do Ceará - UFC. Campus do Pici, Bloco 807, CEP 60455760 Fortaleza (CE). Bolsista produtividade de pesquisa do CNPq. E-mail: mailto:teo@ufc.br

(5) Professor do Departamento de Solos, UFV. Bolsista produtividade de pesquisa do CNPq. E-mail: esm@ufv.br

${ }^{(6)}$ Pesquisador da Embrapa Caprinos. Estrada Sobral/Groaíras, km 4, zona rural, CEP 62010-970 Sobral (CE). E-mail: ambrosio@cnpc.embrapa.br
} 
O tratamento SILV preservou e, em alguns casos, aumentou os teores de $\mathbf{N}$ do solo e, portanto, constituiu um sistema que pode ser recomendado como uma alternativa sustentável de manejo do solo para o semi-árido cearense.

Termos de indexação: nitrogênio mineral, nitrogênio microbiano, substâncias húmicas, semi-árido.

\section{SUMMARY: NITROGEN FRACTIONS IN A LUVISOL UNDER AGROFORESTRY AND CONVENTIONAL SYSTEMS IN THE SEMI-ARID ZONE OF CEARÁ, BRAZIL}

The agroforestry systems have been widely promoted as one sustainable agricultural system, mainly for developing areas, where the external use of resources are not feasible. The aim of this study was to evaluate the impact of one conventional and four agroforestry systems on the contents of total soil nitrogen, mineral nitrogen and different organic nitrogen fractions after five years of experimental cultivation on a Luvisol (Alfisol). The study area was in the semi-arid zone at the National Caprine Research Center (CNPC) of Embrapa, Ceará, Brazil. The following treatments were tested: agrosilvopasture (AGP), silvopasture (SILV), traditional agroforestry in 1998 and 1999 (TR98), traditional agroforestry in 2002 (TR02), conventional cropping (CC), and seasonally dry native vegetation ("Caatinga") (CA). Total- $\mathrm{N}, \mathrm{N}-\mathrm{NH}_{4}^{+}$, $\mathrm{N}$ - $\mathrm{NO}_{3}^{-}$, microbial- $\mathrm{N}$, light organic matter $\mathrm{N}$ (free and occluded) and $\mathrm{N}$ in the humic substances were evaluated. Results indicated high nitrate- $\mathrm{N}\left(\mathrm{NO}_{3}^{-}\right)$content in all treatments, accounting for 10.3 to $23.5 \%$ of the total $N$. The CC treatment reduced the total $N$ and $N$ in humic substances by 38 and $44 \%$, respectively. The AGP and TR98 agroforestry treatments significantly reduced $N$ in the light organic matter and in humic substances. On the other hand, the SILV system preserved, and in some cases, improved the concentration of the soil. This system can therefore be recommended as a sustainable alternative for soil management in the semi-arid region of Ceará state.

Index terms: mineral nitrogen, microbial nitrogen, humic substances, semi-arid, silvopasture.

\section{INTRODUÇÃ̃o}

A região semi-árida brasileira é caracterizada por altas temperaturas, reduzida pluviosidade, solos pouco intemperizados e pequena produção de fitomassa. Somando-se a essas condições edafoclimáticas, observase o predomínio de sistemas agropecuários basicamente extrativistas. Na pecuária, predomina o superpastoreio. A agricultura é desenvolvida à custa de desmatamento indiscriminado, queimadas e períodos de pousio inadequados. Há ainda intensa extração de lenha e madeira para atender à demanda familiar e comercial de cerâmicas e padarias, contribuindo para redução da vegetação da Caatinga (Araújo Filho, 2002). A soma desses fatores faz das regiões semi-áridas ambientes extremamente frágeis, tanto do ponto de vista ambiental quanto socioeconômico (Breman \& Kessler,1997; Sánchez, 2001; Sharma et al., 2005).

Nesse contexto, o solo é considerado um recurso natural essencial, e sua qualidade é responsável não somente pelas boas produtividades da agropecuária, mas também pela manutenção da qualidade do meio ambiente e, conseqüentemente, pela sanidade de plantas, animais e seres humanos (Sharma et al., 2005).

Na região semi-árida brasileira, onde a maior parte da agropecuária se baseia na pequena propriedade, o uso de insumos agrícolas, como adubos nitrogenados, calcário e outros, torna-se geralmente inviável, em razão da baixa disponibilidade de capital por parte dos agricultores. Assim, o entendimento da ciclagem natural dos nutrientes, principalmente o $\mathrm{N}$, é fundamental para o desenvolvimento de uma agricultura sustentável.

Para avaliação da viabilidade ambiental de um determinado agrossistema, tem sido sugerido como ferramenta o monitoramento da qualidade do solo (D’Andrea et al., 2002; Sharma et al., 2005). A matéria orgânica do solo (MOS), ou, mais precisamente, seu conteúdo de $\mathrm{Ce}$, ou, $\mathrm{N}$, é considerada um dos principais indicadores de sua qualidade (Lal, 1997; Mielniczuk, 1999), o que se deve, primeiramente, à sua sensibilidade em relação às práticas de manejo do solo, sobretudo nas regiões tropicais, e, segundo, à sua interação com praticamente todos os processos que ocorrem no solo (Silva \& Resck, 1997; D'Andrea et al., 2002).

O estoque da MOS é regulado principalmente pelo aporte dos resíduos orgânicos e pela taxa de decomposição das frações orgânicas. Vários são os estudos que evidenciam que a substituição da vegetação nativa por sistemas agrícolas altera tanto a dinâmica de aporte de resíduos orgânicos quanto 
sua taxa de decomposição (Haynes, 1999; Chan et al., 2002; Zinn et al., 2005). Em geral, os sistemas agrícolas convencionais são caracterizados pelo maior revolvimento do solo, o que pode provocar a redução da estabilidade estrutural deste (Maia et al., 2006; Green et al., 2007), expondo a MOS à maior ação da microbiota, resultando na diminuição nos teores de MOS (Solomon et al., 2000; Zibilske et al., 2002; Sharma et al., 2005). Inversamente, sistemas mais conservacionistas, que priorizam o aporte de resíduos orgânicos e a menor mobilização do solo, como o plantio direto (Wright et al., 2005), os sistemas agroflorestais (Kaur et al., 2000) e silvipastoris (Maia et al., 2006) e os cultivos orgânicos (Xavier et al., 2006), têm se mostrado eficientes em manter e, às vezes, elevar os teores de $\mathrm{N}$ e $\mathrm{C}$ do solo, preservando sua qualidade.

Com o intuito de facilitar o entendimento da natureza dinâmica da MOS e de como esta pode ser influenciada pelas práticas de manejo e pelas mudanças no ambiente do solo, tem-se desenvolvido o conceito de frações da MOS, pressupondo que estas diferem quanto à suceptibilidade à decomposição microbiana e à longevidade no solo (Stevenson \& Cole, 1999; Chan et al., 2002). Quatro frações baseadas na dinâmica da MOS foram propostas por Duxbury et al. (1989): (1) uma fração ativa, constituída pela biomassa microbiana do solo; (2) uma fração lábil, de compostos prontamente oxidáveis; (3) uma fração física ou estruturalmente protegida, muito lentamente oxidada, associada aos agregados do solo; e (4) uma fração passiva ou recalcitrante, sob influência direta da mineralogia da argila, dificilmente alterada pelas práticas de manejo.

Os sistemas agroflorestais (SAFs) têm sido amplamente promovidos como sistemas de produção agrícola sustentáveis e particularmente atraentes para regiões subdesenvolvidas, onde o uso de insumos externos é inviável (Breman \& Kessler, 1997; Schroth et al., 2002). Os SAFs apresentam inúmeras vantagens, que contribuem para o estabelecimento de modelos de produção mais estáveis e que podem amenizar as adversidades encontradas pela agropecuária nas regiões semi-áridas (Franzel et al., 2001; Araújo Filho \& Carvalho, 2001). Esses sistemas proporcionam maior cobertura do solo, favorecem a preservação da fauna e da flora, promovem a ciclagem de nutrientes a partir da ação de sistemas radiculares diversos e propiciam contínuo aporte de resíduos orgânicos (Breman \& Kessler,1997; Araújo et al., 2001; Sánchez, 2001; Schroth et al., 2002).

Considerando os aspectos apresentados, o presente estudo objetivou avaliar o impacto de quatro SAFs e de um sistema convencional sobre os teores de $\mathrm{N}$ total do solo, mineral e dos compartimentos orgânicos no solo, comparativamente à condição natural (Caatinga nativa), após cinco anos de uso na região semi-árida cearense, em experimento instalado sobre um Luvissolo Crômico órtico, no município de Sobral, CE.

\section{MATERIAL E MÉTODOS}

\section{Localização e descrição das áreas experimen- tais}

O estudo foi realizado na Fazenda Crioula, pertencente ao Centro Nacional de Pesquisa de Caprinos - Embrapa Caprinos, situada no município de Sobral - CE. O município está a $3^{\circ} 41^{\prime} \mathrm{S}$ e $40^{\circ}$ $20^{\prime} \mathrm{W}$, com altitude de $69 \mathrm{~m}$. O clima é tropical equatorial, seco, muito quente e semi-árido do tipo BSW'h, de acordo com a classificação de Köppen (Brasil, 1981). A média anual de precipitação é de $798 \mathrm{~mm}$, com predomínio de estações secas com sete a oito meses de duração (junho a janeiro) e uma estação chuvosa de fevereiro a maio. O solo dominante é um Luvissolo Crômico órtico (Embrapa, 1999) (Quadro 1).

Os sistemas avaliados foram escolhidos a partir de projeto de pesquisa desenvolvido na Embrapa Caprinos desde 1997, que tem como objetivo avaliar três sistemas agrossilvipastoris de produção: o tradicional, o agrossilvipastoril fixo e o agrossilvipastoril rotativo. O sistema tradicional consiste de uma área de 8,0 ha dividida em 10 subparcelas de 0,8 ha, onde anualmente uma das subparcelas é submetida às práticas da agricultura itinerante, ou seja, faz-se o corte e a queima da vegetação, com posterior plantio de milho por dois anos consecutivos, e, em seguida, a subparcela é deixada em pousio. Os 7,2 ha restantes são utilizados como área de mantença de um rebanho de ovinos. No sistema agrossilvipastoril rotativo, uma área de 8,0 ha foi dividida em três subparcelas de 1,6, 4,8 e 1,6 ha, destinadas, respectivamente, à agricultura (agrossilvipastoril), pecuária (silvipastoril) e Caatinga (reserva legal). Nesse contexto, as áreas silvipastoril e de Caatinga constituem os piquetes de mantença de um rebanho de 20 matrizes ovinas, enquanto a área cultivada serve como banco de proteínas durante o período seco. Está previsto para este sistema uma rotação entre as subparcelas após um período de ocupação de sete anos. O sistema agrossilvipastoril fixo segue o mesmo delineamento do sistema rotativo, e não há rotação entre as subparcelas. Avaliou-se também um sistema de cultivo convencional.

Considerando os sistemas citados, foram selecionados e estudados os sistemas: Tratamento Agrossilvipastoril (AGP): é oriundo do modelo rotativo, que adotou o cultivo em aléias com faixas de 3,0 $\mathrm{m}$ de largura, cultivando-se milho intercalado por fileiras de leucena, implantadas com espaçamento de 0,5 m entre plantas. Para o estabelecimento das culturas, a área de 1,6 ha teve a sua vegetação natural rareada, preservando-se uma cobertura vegetal arbórea nativa de $22 \%$, o que equivale a, aproximadamente, 200 árvores ha-1. Tratamento Silvipastoril (SILV): este tratamento foi também oriundo do modelo rotativo e consistiu de uma área de 4,8 ha, submetida ao processo de rareamento, preservandose aproximadamente 260 árvores ha-1, o que 
Quadro 1. Caracterização físico-química do solo da camada de $0-40 \mathrm{~cm}$, nos sistemas agroflorestais e convencional

\begin{tabular}{|c|c|c|c|c|c|c|}
\hline \multirow{2}{*}{ Atributo } & \multicolumn{6}{|c|}{ Tratamento $^{(1)}$} \\
\hline & AGP & SILV & TR98 & TR02 & $\mathrm{CC}$ & CA \\
\hline Argila $\left(\mathrm{g} \mathrm{kg}^{-1}\right)$ & 113,9 & 113,4 & 164,4 & 249,6 & 139,7 & 168,4 \\
\hline Areia grossa $\left(\mathrm{g} \mathrm{kg}^{-1}\right)$ & 265,7 & 461,6 & 308,6 & 286,0 & 318,4 & 321,6 \\
\hline Areia fina $\left(\mathrm{g} \mathrm{kg}^{-1}\right)$ & 364,7 & 216,2 & 295,1 & 206,0 & 305,0 & 233,3 \\
\hline Silte $\left(\mathrm{g} \mathrm{kg}^{-1}\right)$ & 255,7 & 208,8 & 233,9 & 258,4 & 236,9 & 276,7 \\
\hline C orgânico (dag kg $\left.{ }^{-1}\right)$ & 1,43 & 1,99 & 1,66 & 2,17 & 1,32 & 2,18 \\
\hline $\mathrm{N}$ total $\left(\right.$ dag $\left.\mathrm{kg}^{-1}\right)$ & 0,17 & 0,19 & 0,20 & 0,24 & 0,12 & 0,19 \\
\hline Relação C/N & 8,40 & 10,5 & 8,30 & 9,00 & 11,0 & 11,4 \\
\hline Densidade $\left(\mathrm{kg} \mathrm{dm}^{-3}\right)$ & 1,59 & 1,65 & 1,68 & 1,58 & 1,64 & 1,63 \\
\hline $\mathrm{Ca}^{2+}\left(\mathrm{cmol}_{\mathrm{c}} \mathrm{dm}^{-3}\right)$ & 21,8 & 6,30 & 16,9 & 20,8 & 14,4 & 22,1 \\
\hline $\mathrm{Mg}^{2+}\left(\mathrm{cmol}_{\mathrm{c}} \mathrm{dm}^{-3}\right)$ & 4,30 & 3,00 & 5,50 & 7,80 & 3,70 & 9,70 \\
\hline $\mathrm{K}^{+}\left(\mathrm{cmol}_{\mathrm{c}} \mathrm{dm}^{-3}\right)$ & 0,90 & 0,85 & 1,20 & 1,10 & 0,70 & 1,30 \\
\hline $\mathrm{Na}^{+}\left(\mathrm{cmol}_{\mathrm{c}} \mathrm{dm}^{-3}\right)$ & 0,20 & 0,08 & 0,20 & 0,80 & 0,15 & 0,20 \\
\hline $\mathrm{CTC}\left(\mathrm{cmol}_{\mathrm{c}} \mathrm{dm}^{-3}\right)$ & 28,0 & 12,7 & 26,1 & 31,7 & 20,7 & 35,6 \\
\hline $\mathrm{pH}$ em $\mathrm{H}_{2} \mathrm{O}$ & 7,00 & 6,40 & 6,80 & 7,00 & 6,80 & 6,80 \\
\hline
\end{tabular}

(1) AGP: agrossilvipastoril; SILV: silvipastoril; TR98: tradicional de 1998; TR02: tradicional de 2002; CC: cultivo convencional; CA: Caatinga.

correspondeu a $38 \%$ de cobertura. Sistema Agrossilvipastoril Tradicional - 1998 (TR98): este sistema é proveniente do sistema tradicional, onde uma área de 0,8 ha foi submetida ao desmatamento e queima dos restolhos lenhoso e folhoso no ano de 1997; a parcela foi cultivada com milho em 1998 e 1999 e, em seguida, deixada em pousio. Sistema Agrossilvipastoril Tradicional - 2002 (TR02): área proveniente do sistema tradicional que foi submetida ao desmatamento e queima da vegetação lenhosa em 2001, sendo cultivada com milho em 2002, ano em que foi realizada a coleta de solo. Cultivo convencional (CC): este sistema corresponde ao convencional, onde uma parcela de 1,0 ha foi desmatada e queimada em 1997 e cultivada com milho de 1998 a 2002 , sem que houvesse nenhum tipo de fertilização do solo. Caatinga (CA): área de Caatinga nativa do sistema rotativo, sendo considerada como testemunha e utilizada no período seco como área de mantença dos animais.

\section{Amostragem de solo}

A amostragem de solo foi realizada em setembro de 2002 e, neste ano, a precipitação anual na fazenda experimental foi de $905 \mathrm{~mm}$; o período chuvoso se encerrou no mês de junho com $61,2 \mathrm{~mm}$, portanto, no momento da coleta o solo apresentava baixa disponibilidade de água. Cinco minitrincheiras (repetições) foram abertas em cada sistema, e as amostras de solo foram coletadas nas profundidades de 0-6 e 6-12 cm. Após secagem em casa de vegetação por $72 \mathrm{~h}$, as amostras foram destorroadas, passadas em peneira de $2 \mathrm{~mm}$ (TFSA) e armazenadas até realização das análises.

\section{Análises químicas e microbiológicas}

$\mathrm{O} \mathrm{N}$ total (Nt) foi obtido a partir da soma do $\mathrm{N}$ obtido a partir de digestão sulfúrica, seguida de destilação de Kjeldahl, conforme método descrito por Tedesco et al. (1995), com o N-NO ${ }_{3}{ }^{-}$quantificado por colorimetria, de acordo com Yang et al.(1998). O $\mathrm{N}-\mathrm{NH}_{4}{ }^{+}$foi quantificado por colorimetria, pelo método descrito por Kempers \& Zweers (1986).

Para extração do $\mathrm{N}$ da biomassa microbiana do solo (Nmic), empregou-se o método de irradiação e extração, conforme Islam \& Weil (1998) e Ferreira et al. (1999), que tem como princípio a irradiação de amostras de solo, utilizando-se forno de microondas de potência igual a $900 \mathrm{~W}$, com o objetivo de provocar o rompimento das células microbianas. O teor de Nmic foi obtido pela diferença entre $\mathrm{N}$ extraído em amostras irradiadas e não-irradiadas, considerando-se o fator de conversão Kn igual a 0,54 (Brookes et al., 1985). A extração do $\mathrm{N}$ foi feita por meio de digestão sulfúrica seguida de destilação Kjeldahl, conforme Tedesco et al. (1995).

A fração leve-livre (FLl) da MOS foi determinada por flotação em solução de NaI com densidade de 
$1,8 \mathrm{~kg} \mathrm{~L}^{-1}$ e centrifugação a $3.200 \mathrm{rpm}$ por 5 min (Sohi et al., 2001). A fração fisicamente protegida, denominada fração leve intra-agregados (FLi), foi determinada a partir do material remanescente no tubo após extração da FLl. Esse material foi submetido à agitação vertical por $16 \mathrm{~h}$, com o objetivo de promover a desagregação do solo e a liberação da matéria orgânica intra-agregados. Em seguida, repetiu-se o procedimento descrito por Sohi et al. (2001). Os teores de N da FLl e da FLi foram quantificados por combustão via seca, em analisador elementar Perkin Elmer CHNS/O 2400.

A fração passiva da MOS foi determinada pelo fracionamento das substâncias húmicas, o qual foi realizado seguindo a técnica da solubilidade diferencial em meio ácido ou alcalino das frações correspondentes, separando-se a fração ácidos fúlvicos (FAF), a fração ácidos húmicos (FAH) e a humina (HUM), de acordo com os conceitos de frações húmicas estabelecidos pela Sociedade Internacional de Substâncias Húmicas (Swift, 1996). A determinação de $\mathrm{N}$ das frações húmicas foi realizada por meio de digestão em meio sulfúrico, seguida de destilação Kjeldahl, conforme Tedesco et al. (1995). Para quantificação de $\mathrm{N}$ nas frações $\mathrm{AF}$ e $\mathrm{AH}$, retirou-se uma alíquota de $10 \mathrm{~mL}$ dos extratos obtidos no fracionamento, enquanto para a fração HUM utilizou-se 1,0 g de material.

\section{Análise estatística}

O delineamento experimental considerado foi blocos ao acaso, com parcelas subdivididas em profundidade e cinco repetições coletadas dentro de cada parcela experimental. Foram considerados como efeitos primários e secundários os sistemas de manejo e as profundidades de coleta de solo, respectivamente. Os dados foram submetidos à análise de variância e o teste de comparação de médias foi o $\mathrm{t}(\mathrm{p}<0,1)$, utilizando-se o programa SAS (Statistical Analysis System) versão 9.0.

\section{RESULTADOS E DISCUSSÃO}

\section{N total}

Os teores de $\mathrm{N}$ total (Nt), em geral, foram mais elevados na camada de $0-6 \mathrm{~cm}$, decrescendo com o aumento da profundidade do solo em todos o sistemas (Quadro 2). O sistema CC apresentou os menores teores de Nt, diferindo $(p<0,1)$ dos sistemas SILV, TR02 e CA na camada de 0-6 cm, o que significou, em cinco anos, redução de $38,7 \%$, em relação ao sistema testemunha (CA) (Quadro 2). O solo sob o sistema TR02 apresentou os maiores teores de Nt, diferindo $(\mathrm{p}<0,1)$ do sistema CA na primeira camada.

A redução do teor de $\mathrm{Nt}$ no sistema $\mathrm{CC}$ foi condizente com os resultados encontrados por Solomon et al. (2000), os quais observaram a perda de $50 \%$ de
$\mathrm{N}$ em uma área cultivada convencionalmente, comparada à vegetação nativa em um Luvissolo Crômico na região semi-árida da Tanzânia. A perda de Nt no sistema CC está provavelmente relacionada ao maior revolvimento do solo provocado pelos cinco anos de cultivo consecutivos. Vários estudos apontam correlação negativa entre revolvimento do solo e perda de N (Solomon et al., 2000; Smith et al., 2001; Zibilske et al., 2002; Mielniczuk et al., 2003). Sistemas mais intensivos promovem a desagregação do solo, expondo a matéria orgânica, o que favorece a oxidação biológica e o aumento da aeração e, sobretudo, da temperatura do solo, que é um dos principais catalisadores dos processos de denitrificação e amonificação (Stevenson \& Cole, 1999).

Outro aspecto que pode ter contribuído com as diferenças encontradas entre os sistemas é a granulometria (Quadro 1). O sistema TR02, por exemplo, apresentou o maior teor de $\operatorname{argila~}\left(250 \mathrm{~g} \mathrm{~kg}^{-1}\right)$ e também os maiores teores de $\mathrm{Nt}$, assim como das frações da matéria orgânica, evidenciando relação entre as frações orgânicas e mineral (Stevenson \& Cole, 1999).

\section{N mineral}

$\mathrm{O}$ sistema CC apresentou teor mais elevado de $\mathrm{N}$ $\mathrm{NH}_{4}{ }^{+}$na camada superficial, diferindo dos demais sistemas (Quadro 2). Já na camada de 6-12 cm houve diferença significativa apenas entre os sistemas TR98 e CC, este último apresentando o maior teor de $\mathrm{N}$ $\mathrm{NH}_{4}{ }^{+}$. Quanto aos teores de N-NO ${ }_{3}{ }^{-}$, o sistema TR02 superou $(\mathrm{p}<0,1)$ os sistemas AGP, SILV e TR98 nas duas camadas avaliadas, seguido pelo sistema CA, que também foi superior ( $p<0,1$ ) aos demais (Quadro 2).

De modo geral, observou-se que o teor de $\mathrm{N}-\mathrm{NH}_{4}{ }^{+}$ aumentou com o acréscimo da profundidade em todos os sistemas, embora a diferença significativa tenha sido verificada apenas no sistema TR98 (Quadro 2). Por outro lado, o N-NO ${ }_{3}{ }^{-}$apresentou comportamento inverso, diminuindo com a profundidade, com diferenças significativas entre os sistemas AGP, SILV, $\mathrm{CC}$ e CA (Quadro 2). Estes resultados evidenciam que a camada superficial do solo apresentou condições favoráveis ao processo de nitrificação, que consiste na transformação do $\mathrm{N}$ amoniacal em $\mathrm{N}$ nítrico, sendo esse processo mediado pela microbiota do solo e influenciado pelos fatores que afetam sua atividade, incluindo o $\mathrm{pH}$ (Quadro 1), cujos valores se encontram em níveis ótimos à nitrificação em todos os sistemas (Camargo et al., 1999; Stevenson \& Cole, 1999).

A participação do $\mathrm{N}$ mineral $\left(\mathrm{N}-\mathrm{NH}_{4}{ }^{+}+\mathrm{N}^{-} \mathrm{NO}_{3}{ }^{-}\right)$ no teor do Nt do solo variou entre $10,7 \%$ no sistema TR98 e 23,5 \% no sistema CC na camada de 0-6 cm. $\mathrm{Na}$ segunda camada, a variação foi de 12,7 a 23,6 \% nos sistemas TR98 e TR02, respectivamente. Os valores das formas minerais de $\mathrm{N}$ demonstraram alta participação da fração mineral no teor de Nt, quando comparado com outros estudos. Segundo Camargo et al. (1999) e Havlin et al. (2005), as formas de 
Quadro 2. Teores de nitrogênio total (Nt), N-NH ${ }_{4}^{+}, \mathrm{N}-\mathrm{NO}_{3}{ }^{-}$e proporções dos teores de $\mathrm{N}_{-} \mathrm{NH}_{4}{ }^{+}$e $\mathrm{N}-\mathrm{NO}_{3}{ }^{-}$nos teores totais de nitrogênio $\left(\mathrm{N}-\mathrm{NH}_{4}^{+} / \mathrm{Nt}\right.$ e $\mathrm{N}-\mathrm{NO}_{3}{ }^{-} / \mathrm{Nt}$ ) nas camadas de 0-6 e 6-12 cm, nos sistemas agroflorestais e convencional, após cinco anos de exploração

\begin{tabular}{|c|c|c|c|c|c|}
\hline Sistema ${ }^{(1)}$ & $\mathrm{Nt}$ & $\mathrm{N}-\mathrm{NH}_{4}{ }^{+}$ & $\mathrm{N}-\mathrm{NO}_{3}{ }^{-}$ & $\mathrm{N}-\mathrm{NH}_{4}{ }^{+} / \mathrm{Nt}$ & $\mathrm{N}-\mathrm{NO}_{3}{ }^{-} / \mathrm{Nt}$ \\
\hline & & $-\mathrm{g} \mathrm{dm}^{-3}$ & & 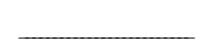 & 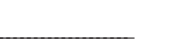 \\
\hline & & & $0-6 \mathrm{~cm}$ & & \\
\hline AGP & $3,76 \mathrm{BCa}$ & $0,0021 \mathrm{Ba}$ & 0,46 CDa & $0,055 \mathrm{AB} b$ & $12,2 \mathrm{Ba}$ \\
\hline SILV & $4,45 \mathrm{ABa}$ & $0,0022 \mathrm{Ba}$ & $0,48 \mathrm{BCDa}$ & $0,049 \mathrm{~B} \mathrm{~b}$ & $10,8 \mathrm{Ba}$ \\
\hline TR98 & $3,77 \mathrm{BCa}$ & $0,0008 \mathrm{Bb}$ & $0,39 \mathrm{Da}$ & $0,021 \mathrm{~B} \mathrm{~b}$ & $10,3 \mathrm{Ba}$ \\
\hline TR02 & $5,46 \mathrm{Aa}$ & $0,0012 \mathrm{Ba}$ & $0,69 \mathrm{Aa}$ & $0,022 \mathrm{~B} \mathrm{~b}$ & $12,6 \mathrm{Bb}$ \\
\hline $\mathrm{CC}$ & $2,45 \mathrm{Ca}$ & $0,0066 \mathrm{Aa}$ & $0,55 \mathrm{ABCa}$ & $0,27 \mathrm{Aa}$ & $22,4 \mathrm{Aa}$ \\
\hline \multirow[t]{2}{*}{$\mathrm{CA}$} & $4,00 \mathrm{Ba}$ & $0,0014 \mathrm{Ba}$ & $0,62 \mathrm{ABa}$ & $0,035 \mathrm{Ba}$ & $15,5 \mathrm{Ba}$ \\
\hline & & & $6-12 \mathrm{~cm}$ & & \\
\hline AGP & $2,22 \mathrm{ABb}$ & $0,0025 \mathrm{BCa}$ & $0,32 \mathrm{Cb}$ & $0,11 \mathrm{ABa}$ & $14,4 \mathrm{BCa}$ \\
\hline SILV & $2,20 \mathrm{ABb}$ & $0,0040 \mathrm{ABCa}$ & $0,27 \mathrm{Cb}$ & $0,18 \mathrm{ABa}$ & $12,3 \mathrm{Ca}$ \\
\hline TR98 & $2,78 \mathrm{Ab}$ & $0,0013 \mathrm{Ca}$ & $0,35 \mathrm{Ca}$ & $0,047 \mathrm{Ba}$ & $12,6 \mathrm{Ca}$ \\
\hline TR02 & $3,02 \mathrm{Ab}$ & $0,0041 \mathrm{ABCa}$ & $0,71 \mathrm{Aa}$ & $0,13 \mathrm{Aba}$ & $23,5 \mathrm{Aa}$ \\
\hline $\mathrm{CC}$ & $1,59 \mathrm{Bb}$ & $0,0044 \mathrm{ABa}$ & $0,31 \mathrm{Cb}$ & $0,28 \mathrm{Aa}$ & $19,5 \mathrm{Aba}$ \\
\hline $\mathrm{CA}$ & $2,50 \mathrm{ABb}$ & $0,0033 \mathrm{BCa}$ & $0,49 \mathrm{Bb}$ & $0,13 \mathrm{ABa}$ & $19,6 \mathrm{Aba}$ \\
\hline
\end{tabular}

\footnotetext{
(1) AGP: agrossilvipastoril; SILV: silvipastoril; TR98: tradicional de 1998; TR02: tradicional de 2002; CC: cultivo convencional; CA: Caatinga. Médias seguidas de letras maiúsculas iguais na coluna dentro da mesma profundidade e minúsculas na coluna entre profundidades dentro do sistema não diferem entre si pelo teste $t(p<0,1)$.
}

$\mathrm{N}$ mineral contribuem com 2 a $5 \%$ do $\mathrm{Nt}$ do solo. Estes resultados elevados estão refletindo, provavelmente, uma série de fatores que contribuíram para a mineralização e manutenção do $\mathrm{N}$ mineral no solo, como altas temperaturas, baixa relação $\mathrm{C} / \mathrm{N}$ (Quadro 1), eficiente ciclagem de nutrientes e, principalmente, época da coleta das amostras de solo. Considerando este último fator, ressalta-se que as amostras foram coletadas no mês de setembro, período seco da região, o que permite inferir que houve utilização mínima de $\mathrm{N}$ mineral pelas plantas, bem como, reduzidas perdas por erosão e, ou, lixiviação, dada a escassez de água no sistema e, ainda, devido

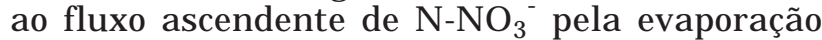
superficial da água.

\section{N da biomassa microbiana do solo}

$\mathrm{O} N$ da biomassa microbiana (Nmic) representa a fração ativa da MOS e está diretamente relacionado com os processos de mineralização e imobilização do $\mathrm{N}$ do solo; ele não apresentou diferenças significativas entre os sistemas na camada de $0-6 \mathrm{~cm}$ e também entre as profundidades (Figura 1). No entanto, todos os sistemas apresentaram teores de Nmic superiores ao observado no sistema CA, na camada de $0-6 \mathrm{~cm}$, o que pode ser devido ao efeito dos SAFs, no que se refere ao maior aporte e diversidade de resíduos orgânicos, resultando na formação de uma MOS de melhor qualidade, que exerce influência sobre a população microbiana (Kaur et al., 2000).

Os sistemas SILV, TR02 e CC, onde os efeitos foram mais pronunciados, apresentaram acréscimo nos

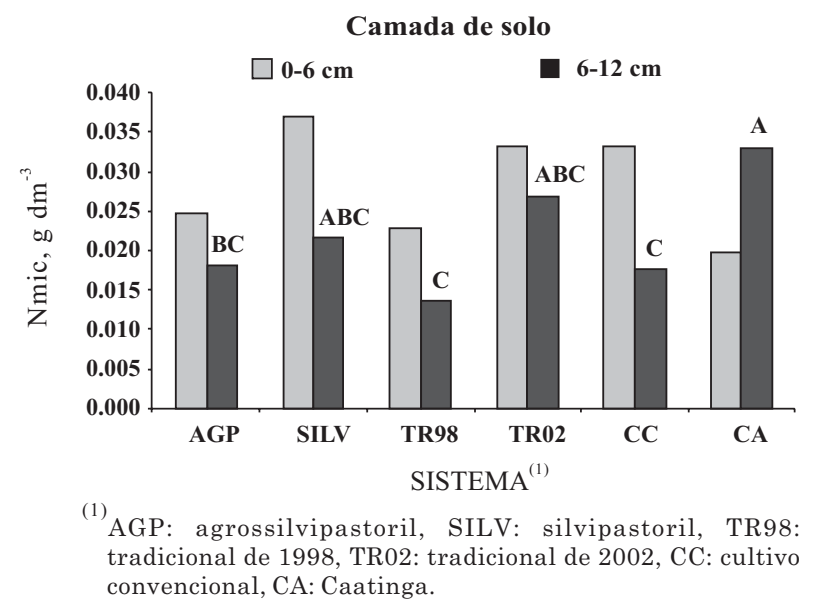

Figura 1. Teores do nitrogênio microbiano (Nmic) nas camadas de 0-6 e 6-12 cm, nos sistemas agroflorestais e convencional, após cinco anos de exploração. Médias seguidas de letras iguais não diferem entre si pelo teste $t(p<0,1)$. 
teores de Nmic de 86, 68 e $68 \%$, respectivamente, em relação ao observado no sistema $\mathrm{CA}$, na camada de 0 $6 \mathrm{~cm}$. Já na camada de solo de 6-12 cm o sistema testemunha (CA) apresentou o maior teor de Nmic, diferindo $(\mathrm{p}<0,1)$ dos sistemas AGP, TR98 e CC, sendo o único sistema em que o teor de Nmic na profundidade de 6-12 cm foi superior ao encontrado em superfície (Figura 1).

No sistema SILV, o maior teor de Nmic deve-se, provavelmente, ao maior aporte de resíduos orgânicos em superfície, proveniente inicialmente do rareamento da área e, em seguida, das podas do estrato arbóreo e, sobretudo, do aumento do estrato herbáceo. Esta hipótese pode ser justificada pelos elevados teores de matéria orgânica leve (Quadro 3), reconhecida como principal fonte de $\mathrm{C}, \mathrm{N}$ e energia para os microrganismos do solo (Silva \& Resck, 1997; D’Andréa et al., 2002). No sistema TR02, a atividade microbiana pode ter sido favorecida pela maior disponibilidade de nutrientes em decorrência da queima da vegetação, mesmo que esse efeito seja temporário, uma vez que a tendência é de que a fertilidade do solo, nessas condições, diminua em curto prazo. Já o sistema CC apresentou o teor de Nmic aumentado em relação à CA na camada superficial $(0-6 \mathrm{~cm})$, o que se deve, provavelmente, aos efeitos das gramíneas que se estabeleceram na parcela durante o período de pousio (seis meses). Além do aporte de resíduos orgânicos em superfície, as gramíneas promovem a liberação de exsudatos (Silva \& Mielniczuk, 1997a) ricos em carboidratos e proteínas e que estão mais disponíveis para os microrganismos (Silva \& Mielniczuk, 1997b).

É importante ressaltar que a avaliação dos resultados de Nmic deve ser criteriosa, uma vez que uma alta atividade biológica pode ser resultado tanto de um grande reservatório de $\mathrm{C}$ e $\mathrm{N}$ lábeis, em que a decomposição é intensa (por exemplo, áreas de vegetação nativa), como da oxidação da MO proveniente da quebra de agregados pela ação antrópica, ou, ainda, como resultado da adição momentânea de resíduos orgânicos. Assim, altos teores de N microbiano podem indicar tanto uma situação de distúrbio quanto uma alta produtividade do ecossistema (Islam \& Weil, 2000).

A participação do Nmic em relação aos teores de Nt variou de 0,49 a $1,35 \%$ na camada de $0-6 \mathrm{~cm}$ e de 0,49 a 1,32 \% na camada de 6-12 cm (Figura 2). Com exceção do sistema TR98, a participação do Nmic no Nt aumentou com o aumento da profundidade, o que provavelmente se deve mais à redução do $\mathrm{Nt}$ na segunda camada do que propriamente ao aumento do Nmic (Figura 1). Na camada superficial, o Nmic representou 1,35\% do Nt no sistema CC. Este valor é mais que o dobro do observado na Caatinga (CA), porém pode ser explicado pela redução significativa do Nt no sistema CC (Quadro 2).

Quadro 3. Teores da fração leve-livre (FLl), leve intra-agregados (FLi), N-FLl e N-FLi nas camadas de 0-6 e 6-12 cm, nos tratamentos agroflorestais e convencional, após cinco anos de exploração

\begin{tabular}{|c|c|c|c|c|}
\hline Sistema $^{(1)}$ & FLl & FLi & N-FLl & N-FLi \\
\hline & \multicolumn{4}{|c|}{$-\mathrm{g} \mathrm{dm}^{-3}$} \\
\hline & \multicolumn{4}{|c|}{$0-6 \mathrm{~cm}$} \\
\hline AGP & $26,3 \mathrm{Ca}$ & $1,36 \mathrm{Bb}$ & $0,331 \mathrm{BCa}$ & $0,0109 \mathrm{C}$ \\
\hline SILV & $38,2 \mathrm{ABCa}$ & $3,64 \mathrm{Aa}$ & $0,545 \mathrm{ABa}$ & $0,0466 \mathrm{Aa}$ \\
\hline TR98 & $20,6 \mathrm{Ca}$ & $1,97 \mathrm{Ba}$ & $0,217 \mathrm{Ca}$ & $0,0167 \mathrm{Ca}$ \\
\hline TR02 & $49,6 \mathrm{Aa}$ & $2,43 \mathrm{Aba}$ & $0,601 \mathrm{Aa}$ & $0,0147 \mathrm{Ca}$ \\
\hline $\mathrm{CC}$ & $28,4 \mathrm{BCa}$ & $3,57 \mathrm{Aa}$ & $0,179 \mathrm{Ca}$ & $0,0231 \mathrm{BCa}$ \\
\hline \multirow[t]{2}{*}{$\mathrm{CA}$} & $47,6 \mathrm{ABa}$ & $3,48 \mathrm{Aa}$ & $0,537 \mathrm{ABa}$ & 0,0332 Ba \\
\hline & \multicolumn{4}{|c|}{$6-12 \mathrm{~cm}$} \\
\hline AGP & $11,2 \mathrm{BCb}$ & $2,54 \mathrm{Aa}$ & $0,074 \mathrm{CDb}$ & $0,0121 \mathrm{~A}$ \\
\hline SILV & $10,2 \mathrm{Cb}$ & $3,67 \mathrm{Aa}$ & $0,081 \mathrm{BCb}$ & $0,0243 \mathrm{Ab}$ \\
\hline TR98 & $13,0 \mathrm{ABCb}$ & $1,63 \mathrm{Aa}$ & $0,099 \mathrm{BCb}$ & $0,0029 \mathrm{Ab}$ \\
\hline TR02 & $17,4 \mathrm{Ab}$ & $3,75 \mathrm{Aa}$ & $0,111 \mathrm{ABb}$ & $0,0061 \mathrm{Ab}$ \\
\hline $\mathrm{CC}$ & $11,0 \mathrm{BCb}$ & $1,29 \mathrm{Ab}$ & $0,042 \mathrm{Db}$ & $0,0035 \mathrm{Ab}$ \\
\hline $\mathrm{CA}$ & $11,4 \mathrm{BCb}$ & $2,60 \mathrm{Ab}$ & $0,080 \mathrm{BCb}$ & $0,0079 \mathrm{Ab}$ \\
\hline
\end{tabular}

\footnotetext{
(1) AGP: agrossilvipastoril; SILV: silvipastoril; TR98: tradicional de 1998; TR02: tradicional de 2002; CC: cultivo convencional; CA: Caatinga. Médias seguidas de letras maiúsculas iguais na coluna dentro da mesma profundidade e minúsculas na coluna entre profundidades dentro de sistemas não diferem entre si pelo teste $\mathrm{t}(\mathrm{p}<0,1)$.
} 


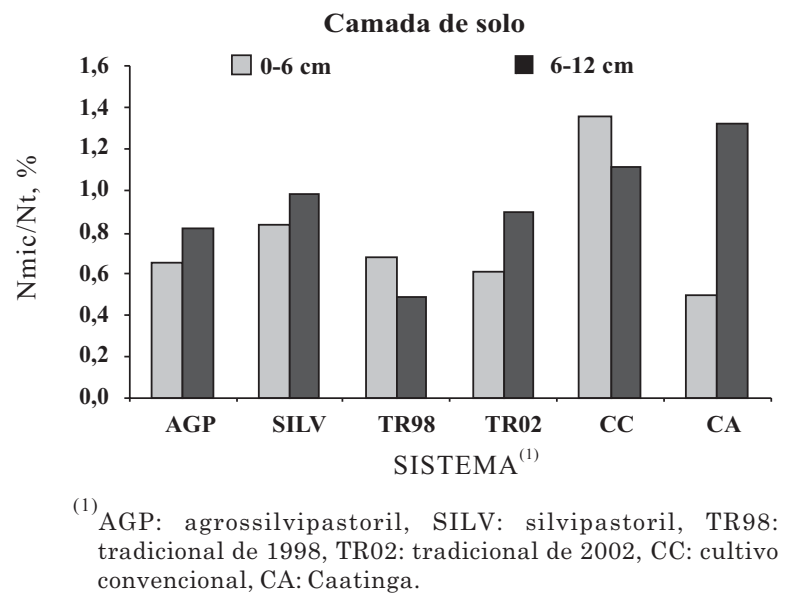

Figura 2. Proporção do Nmic em relação ao $\mathrm{Nt}$ nas camadas de 0-6 e 6-12 cm, nos tratamentos agroflorestais e convencional, após cinco anos de exploração.

Os resultados encontrados para participação do Nmic no Nt estão abaixo dos valores comumente observados na literatura, que geralmente variam entre 2 e $5 \%$ (Stevenson \& Cole, 1999; Souza \& Melo, 2000; D'Andréa et al., 2002). Esses resultados estão relacionados com os teores de $\mathrm{Nt}$, que, em geral, foram elevados (Quadro 2), quando comparados a outros trabalhos em condições ambientais semelhantes (Kaur et al., 2000; Wright et al., 2005; Xavier et al., 2006) e também diferentes (Silva \& Resck, 1997), o que permite inferir que as condições edafoclimáticas da região estudada e os SAFs adotados favoreceram a manutenção do $\mathrm{N}$ no solo e, ou, reduziram a atividade microbiana.

\section{N da fração leve}

A fração leve, devido à sua labilidade, assim como a biomassa microbiana, tem sido utilizada como indicador sensível das alterações ocorridas no solo (Yang \& Kay, 2001; Chan et al., 2002; Xavier et al., 2006). Especificamente no solo, essa fração possui duas funções diretas. A primeira está relacionada com a sua utilização como substrato pela microbiota do solo, configurando-se como a principal fonte energética do meio; e, a segunda, como fonte de nutrientes, principalmente de $\mathrm{N}$, em razão de seus componentes serem prontamente oxidáveis (Stevenson \& Cole, 1999; Haynes, 1999).

Comparativamente ao sistema CA, os sistemas AGP e TR98 apresentaram redução significativa de 45 e $57 \%$, respectivamente, no teor da FLl, na camada de 0-6 cm (Quadro 3). O sistema CC, apesar de não diferir significativamente de $\mathrm{Ca}$, causou diminuição de $40 \%$ na FLl, resultado similar ao encontrado no solo sob o sistema AGP. Na camada de 0-6 cm, o sistema TR02 apresentou o maior conteúdo de FLl, seguido de TR98, diferindo $(p<0,1)$ dos demais sistemas (Quadro 3).
Constatou-se que as principais alterações na FLl ocorreram na camada superficial e nos sistemas que promoveram maior movimentação do solo-neste caso, o AGP, TR98 e CC. Nos sistemas AGP e CC, a redução nos teores de FLl parece estar diretamente relacionada ao revolvimento do solo causado pelos cinco anos de cultivo. Esses resultados são semelhantes aos encontrados em outros estudos (Smith et al., 2001; Zibilske et al., 2002; Mielniczuk et al., 2003), que evidenciam o efeito do revolvimento do solo sobre a perda da matéria orgânica via oxidação biológica. Já no sistema TR98 as perdas da FLl foram geradas, provavelmente, pelo efeito conjunto da queima da vegetação e devido aos dois anos consecutivos de cultivo - resultados similares aos encontrados por Chan et al. (2002).

Os teores de fração leve intra-agregrados (FLi) diferenciaram-se significativamente apenas na camada de 0-6 cm, em que os sistemas AGP e TR98 tiveram seus teores reduzidos em 61 e $43 \%$, respectivamente, quando comparados à área de referência (CA) (Quadro 3). Esses resultados assemelham-se aos encontrados na FLl e confirmam a suceptibilidade do solo destes sistemas à perda da MO. Os teores de FLi na camada de 6-12 cm reforçam essa idéia, pois observou-se menor variação entre os sistemas, indicando o maior efeito do manejo na superfície do solo. Por ser uma fração diretamente relacionada com a formação e estabilização dos agregados do solo, a redução da fração leve da matéria orgânica com o tempo poderá comprometer a sustentabilidade do sistema, atuando principalmente sobre os atributos físicos do solo (Solomon et al., 2000; Chan et al., 2002).

Quanto ao N contido na FLl (N-FLl), os sistemas TR98 e CC reduziram os teores do solo de forma significativa em relação ao sistema $\mathrm{CA}$, na camada de 0-6 cm (Quadro 3). Esses resultados ressaltam os efeitos negativos da mobilização do solo, indicando que estes sistemas ou promoveram a perda de $\mathrm{N}$ da FLl ou propiciaram um aporte de $\mathrm{MO}$ de menor qualidade, com maior relação $\mathrm{C} / \mathrm{N}$. Os comportamentos observados estão de acordo com os publicados em outros estudos (Yang \& Kay, 2001; Chan et al., 2002).

Já os resultados do N-FLi mostraram que os sistemas AGP, TR02 e TR98 promoveram maior redução $(\mathrm{p}<0,1)$ do teor desta fração na camada superficial, comparado com CA (Quadro 3). Quanto ao tratamento AGP, Maia et al. (2006) constataram que houve redução de $26 \%$ nos macroagregados (4,76-2,0 mm), em relação ao sistema de referência CA. Supõe-se que isso tenha provocado a exposição da FLi, com conseqüente perda do N. Por outro lado, os sistemas TR02 e TR98 não apresentaram alterações quanto ao seu estado de agregação (Maia et al., 2006); logo, a redução nos teores de N-FLi deve estar associada com o uso do fogo e, ou, com o aporte de $\mathrm{MO}$ com maior relação $\mathrm{C} / \mathrm{N}$.

De modo geral, os resultados evidenciaram que a MOL e o teor de $\mathrm{N}$ desta fração sofreram as maiores reduções na camada superficial nos sistemas AGP, TR98 e CC, onde houve mobilização do solo. 


\section{N nas substâncias húmicas}

O N das substâncias húmicas representou a maior parte do Nt do solo (Quadro 4), variando na camada húmicas nos sistemas mais intensivos (AGP, TR98 e $\mathrm{CC}$ ) indicam que as condições edafoclimáticas da área experimental e as práticas adotadas nestes tratamentos foram suficientes para atingir não

Quadro 4. Proporções dos teores de N das frações de ácidos fúlvicos (FAF), ácidos húmicos (FAH), humina (HUM) e soma das três frações (FAF + FAH + HUM) em relação aos teores de nitrogênio total (Nt) do solo nas camadas de 0-6 e 6-12 cm, nos sistemas agroflorestais e convencional, após cinco anos de exploração

\begin{tabular}{|c|c|c|c|c|}
\hline Sistema $\mathbf{a}^{(1)}$ & $\mathrm{FAF} / \mathrm{Nt}$ & $\mathrm{FAH} / \mathrm{Nt}$ & HUM/Nt & $\mathrm{FAF}+\mathrm{FAH}+\mathrm{HUM} / \mathrm{Nt}$ \\
\hline & \multicolumn{4}{|c|}{ - $\%$} \\
\hline AGP & $3,5 \mathrm{Ba}$ & $13,8 \mathrm{Ab}$ & $40,7 \mathrm{Ba}$ & 58,0 \\
\hline SILV & $4,5 \mathrm{ABa}$ & $16,6 \mathrm{Ab}$ & $46,1 \mathrm{ABa}$ & 67,2 \\
\hline TR98 & $4,7 \mathrm{ABa}$ & $15,4 \mathrm{Ab}$ & $46,4 \mathrm{ABa}$ & 66,3 \\
\hline TR02 & $5,3 \mathrm{ABb}$ & $25,3 \mathrm{Aa}$ & $54,2 \mathrm{AB} \mathrm{b}$ & 84,8 \\
\hline $\mathrm{CC}$ & $7,8 \mathrm{Aa}$ & $23,3 \mathrm{Ab}$ & $56,7 \mathrm{ABa}$ & 87,8 \\
\hline \multirow[t]{2}{*}{$\mathrm{CA}$} & $7,0 \mathrm{Aa}$ & $21,5 \mathrm{Aa}$ & $69,0 \mathrm{ABa}$ & 97,5 \\
\hline & \multicolumn{4}{|c|}{$6-12 \mathrm{~cm}$} \\
\hline AGP & $5,0 \mathrm{Aa}$ & $17,6 \mathrm{Ba}$ & $43,7 \mathrm{Ba}$ & 66,2 \\
\hline SILV & $8,6 \mathrm{Aa}$ & $41,4 \mathrm{Aa}$ & $36,4 \mathrm{Ba}$ & 86,4 \\
\hline TR98 & $6,5 \mathrm{Aa}$ & $17,3 \mathrm{Ba}$ & $50,7 \mathrm{ABa}$ & 74,5 \\
\hline TR02 & $9,3 \mathrm{Aa}$ & $30,1 \mathrm{Aba}$ & $65,9 \mathrm{Aa}$ & 105,3 \\
\hline $\mathrm{CC}$ & $8,2 \mathrm{Aa}$ & $30,2 \mathrm{Ba}$ & $57,9 \mathrm{ABa}$ & 96,2 \\
\hline $\mathrm{CA}$ & $6,8 \mathrm{Aa}$ & $23,6 \mathrm{Ba}$ & $54,0 \mathrm{ABa}$ & 84,4 \\
\hline
\end{tabular}

(1) AGP: agrossilvipastoril; SILV: silvipastoril; TR98: tradicional de 1998; TR02: tradicional de 2002; CC: cultivo convencional; CA: Caatinga. Médias seguidas de letras maiúsculas iguais na coluna dentro da mesma profundidade e minúsculas na coluna entre profundidades dentro de sistemas não diferem entre si pelo teste $t(p<0,1)$.

de $0-6 \mathrm{~cm}$ entre $58,0 \%$ no sistema AGP e $97,5 \%$ no sistema de referência CA, e na camada de $6-12 \mathrm{~cm}$, entre $66,2 \%$ em AGP e 105,3\% em TR02. É importante ressaltar que o fato de a soma das frações das substâncias húmicas exceder os $100 \%$, no sistema TR02, na camada 6-12 cm, está relacionado com prováveis erros analíticos. Os resultados, sobretudo da camada superficial, demonstraram redução nos teores de substâncias húmicas em todos os sistemas em relação à área de Caatinga (CA), reforçando o efeito da ação antrópica. Observou-se, também, que houve predomínio do teor de N-fração humina (HUM) sobre $\mathrm{N}$-fração ácidos húmicos (FAH) e desta sobre N-fração ácidos fúlvicos (FAF), em todos os sistemas (Quadro 4). Esses resultados são indicativos de que os sistemas apresentaram condições favoráveis ao processo de humificação da matéria orgânica.

Entretanto, os solos sob sistemas que promoveram maior mobilização, como AGP, TR98 e CC, apresentaram, de modo geral, redução significativa nos teores de $\mathrm{N}$ das substâncias húmicas em relação ao sistema CA, sendo esse efeito mais pronunciado na camada de 0-6 cm (Quadro 5).

Apesar de as substâncias húmicas representarem a fração passiva da MOS e serem caracterizadas como a de moléculas orgânicas de maior recalcitrância, ou seja, mais dificilmente alterada pelas práticas de manejo (Duxbury, 1989; Stevenson, 1994; Silva \& Resck, 1997), as reduções observadas nas frações
Quadro 5. Teores de nitrogênio nas frações ácido fúlvico (FAF), ácido húmico (FAH) e humina (HUM) nas camadas de 0-6 e 6-12 cm, nos sistemas agroflorestais e convencional, após cinco anos de exploração

\begin{tabular}{llll}
\hline Sistema $^{(1)}$ & FAF & FAH & HUM \\
& & \multicolumn{3}{c}{ g dm $^{-3}$} \\
\cline { 2 - 4 } & & $0-6 \mathrm{~cm}$ \\
AGP & $0,13 \mathrm{Ca}$ & $0,52 \mathrm{Ca}$ & $1,53 \mathrm{CDa}$ \\
SILV & $0,20 \mathrm{Ba}$ & $0,74 \mathrm{BCa}$ & $2,05 \mathrm{CDa}$ \\
TR98 & $0,17 \mathrm{BCa}$ & $0,58 \mathrm{Ca}$ & $1,75 \mathrm{Aa}$ \\
TR02 & $0,29 \mathrm{Aa}$ & $1,38 \mathrm{Aa}$ & $2,96 \mathrm{Da}$ \\
CC & $0,19 \mathrm{BCa}$ & $0,57 \mathrm{Ca}$ & $1,39 \mathrm{Da}$ \\
CA & $0,28 \mathrm{Aa}$ & $0,86 \mathrm{Ba}$ & $2,76 \mathrm{ABa}$ \\
& & $6-12 \mathrm{~cm}$ & \\
AGP & $0,11 \mathrm{Ba}$ & $0,39 \mathrm{Bb}$ & $0,97 \mathrm{BCb}$ \\
SILV & $0,19 \mathrm{ABa}$ & $0,91 \mathrm{Aa}$ & $0,80 \mathrm{Cb}$ \\
TR98 & $0,18 \mathrm{ABa}$ & $0,48 \mathrm{Bb}$ & $1,41 \mathrm{Bab}$ \\
TR02 & $0,28 \mathrm{Aa}$ & $0,91 \mathrm{Aab}$ & $1,99 \mathrm{Ab}$ \\
CC & $0,13 \mathrm{Bb}$ & $0,48 \mathrm{Bab}$ & $0,92 \mathrm{BCb}$ \\
CA & $0,17 \mathrm{ABb}$ & $0,59 \mathrm{ABb}$ & $1,35 \mathrm{Bb}$ \\
& &
\end{tabular}

(1) AGP: agrossilvipastoril; SILV: silvipastoril; TR98: tradicional de 1998; TR02: tradicional de 2002; CC: cultivo convencional; CA: Caatinga. Médias seguidas de letras maiúsculas iguais na coluna dentro de profundidade e minúsculas na coluna entre profundidades dentro de sistemas não diferem entre si pelo teste $\mathrm{t}(\mathrm{p}<0,1)$. 
somente as frações mais lábeis, como também a fração mais estável da MOS. Esse fato pode estar se refletindo nos altos teores do $\mathrm{N}$ mineral observados nos tratamentos (Quadro 2), o que é um aspecto positivo no que se refere à produtividade agrícola. Contudo, a médio e longo prazo, poderá comprometer a sustentabilidade destes sistemas.

\section{CONCLUSÕES}

1. Os teores de $\mathrm{N}-\mathrm{NO}_{3}{ }^{-}$foram elevados em todos os sistemas estudados.

2. O sistema de cultivo convencional causou, na camada superficial do solo $(0-6 \mathrm{~cm})$, redução nos teores de $\mathrm{N}$ total e das frações das substâncias húmicas em 38,7 e $44,8 \%$, respectivamente, em comparação ao tratamento mata nativa. Esses resultados evidenciam a inviabilidade deste tipo de sistema agrícola para as condições semi-áridas do Ceará.

3. Sistemas agroflorestais que promoveram o maior revolvimento do solo causaram redução dos teores de $\mathrm{N}$ total, $\mathrm{N}$ da fração leve (livre e oclusa) e $\mathrm{N}$ das substâncias húmicas. O sistema SILV representa alternativa sustentável de manejo do solo e deve ser utilizado em sistemas de rotação com outros SAFs na região semi-árida cearense.

\section{AGRADECIMENTOS}

Este trabalho foi financiado pelo Programa de Cooperação Acadêmica (PROCAD) da CAPES, que envolveu as Universidades Federais do Ceará (UFC) e de Viçosa (UFV). Agradecemos ao Sr. Braz Júlio (UFV) pelo auxílio nas análises de solo e ao Centro Nacional de Pesquisas de Caprinos - CNPC/Embrapa por viabilizar o trabalho na Fazenda Crioula.

\section{LITERATURA CITADA}

ARAÚJO FILHO, J.A. Caatinga: Agroecologia versus desertificação. Ci. Hoje, 30:44-45, 2002.

ARAÚJO FILHO, J.A. \& CARVALHO, F.C. Sistemas de produção agrossilvipastoril para o semi-árido nordestino. In: CARVALHO, M.M.; ALVIM, M.J. \& CARNEIRO, J.C., eds. Sistemas agroflorestais pecuários: Opções de sustentabilidade para áreas tropicais e subtropicais. Juiz de Fora, 2001. p.101-110.

ARAÚJO, G.G.L.; ALBUQUERQUE, S.G. \& GUIMARÃES FILHO, C. Opções no uso de forrageiras arbustivoarbóreas na alimentação animal no semi-árido do Nordeste. In: CARVALHO, M.M.; ALVIM, M.J. \& CARNEIRO, J.C., eds. Sistemas agroflorestais pecuários: Opções de sustentabilidade para áreas tropicais e subtropicais. Juiz de Fora, 2001. p.111-137.
BRASIL.Ministério das Minas e Energia. RADAMBRASIL. Folhas SB. 24/25 - Jaguaribe/Natal 1981. Geologia/ Geomorfologia/Pedologia/Vegetação/Uso Potencial da Terra. Rio de Janeiro, 740p. (Levantamento de Recursos Naturais)

BREMAN, H. \& KESSLER, J.J. The potential benefits of agroforetry in the Sahel and other semi - arid regions. Eur. J. Agron., 7:25-33, 1997.

BROOKES, P.C.; LANDMAN, A.; PRUDEN, G. \& JENKINSON, D.S. Chloroform furrigation and the release of soil nitrogen: A rapid direct extraction method for measuring microbial biomass nitrogen in soil. Soil Biol. Biochem., 17:837-842, 1985.

CAMARGO, F.A.O.; GIANELLO, C.; TEDESCO, M.J. \& VIDOR, C. Nitrogênio orgânico do solo. In: SANTOS, G.A. \& CAMARGO, F.A.O. eds. Fundamentos da matéria orgânica do solo. Ecossistemas tropicais e subtropicais. Porto Alegre, Genesis, 1999. p.117-137.

CHAN, K.Y.; HEENAN, D.P. \& OATES, A. Soil carbon fractions and relationship to soil quality under different tillage and stubble management. Soil Till. Res., 63:133-139, 2002.

D‘ANDRÉA, A.F.; SILVA, M.L.N.; CURI, N.; SIQUEIRA, J.O. \& CARNEIRO, M.A.C. Atributos biológicos indicadores da qualidade do solo em sistemas de manejo na região do Cerrado no sul do Estado de Goiás. R. Bras. Ci. Solo, 26:913-923, 2002 .

DUXBURY, J.M.; SMITH, M.S. \& DORAN, J.W. Soil organic matter as a source and a sink of plant nutrients. In: COLEMAN, D.C., ed. Dynamics of soil organic matter in tropical ecosystems. Honolulu, Niftal Project, 1989. p.3367.

EMPRESA BRASILEIRA DE PESQUISA AGROPECUÁRIA EMBRAPA. Centro Nacional de Pesquisa de Solos. Sistema brasileiro de classificação de solos. Brasília, Embrapa Produção de Informação, 1999. 412p.

FERREIRA, A.S.; CAMARGO, F.A.O. \& VIDOR, C. Utilização de microondas na avaliação da biomassa microbiana do solo. R. Bras. Ci. Solo, 23:991-996, 1999.

FRANZEL, S.; COE, R.; COOPER, P.; PLACE, F. \& SCHERR, S.J. Assessing the adoption potential of agroforestry practices in sub-Saharan Africa. Agric. Syst., 69:37-62, 2001

GREEN, V.S.; STOTT, D.E.; CRUZ, D.E. \& CURI, N. Tillage impacts on soil biological activity and aggregation in a Brazilian Cerrado Oxisol. Soil Till. Res., 92:114-121, 2007.

HAVLIN, J.L.; BEATON, J.D.; TISDALE, S.L. \& NELSON, W.B. Soil fertility and fertilizers: An introduction to nutrient management. 7.ed. New Jersey, Pearson Prentice Hall, 2005. 515p.

HAYNES, R.J. Labile organic matter fractions and aggregate stability under short-term, grass-based leys. Soil Biol. Biochem., 31:1821-1830, 1999. 
ISLAM, K.R. \& WEIL, R.R. Land use effects on soil quality in a tropical forest ecosystem of Bangladesh. Agric. Ecosys. Environ., 79:9-16, 2000.

ISLAM, K.R. \& WEIL, R.R. Microwave irradiation of soil for routine measurement of microbial biomass carbon. Biol. Fert. Soils, 27:408-416, 1998.

KAUR, B.; GUPTA, S.R. \& SINGH, G. Soil carbon microbial activity and nitrogen availability in agroforestry systems on moderatily alkaline soils in Northern India. Appl. Soil Ecol., 15:283-294, 2000.

KEMPERS, A.J. \& ZWEERS, A. Ammonium determination in soil extracts by salicylate method. Comm. Soil Sci. Plant Anal., 17:715-723, 1986.

LAL, R. Residue management, conservation tillage and restoration for mitigating greenhouse effect by $\mathrm{CO}_{2}$ enrichment. Soil Till. Res., 43:81-107, 1997.

MAIA, S.M.F.; XAVIER, F.A.S.; OLIVEIRA, T.S.; MENDONÇA, E.S. \& ARAÚJO FILHO, J.A. Impactos de sistemas agroflorestais e convencional sobre a qualidade do solo no semi-árido cearense. R. Árvore, 30:837-848, 2006.

MIELNICZUK, J. Matéria orgânica e a sustentabilidade de sistemas agrícolas. In: SANTOS, G.A. \& CAMARGO, F.A.O., eds. Fundamentos da matéria orgânica do solo. Ecossistemas tropicais e subtropicais. Porto Alegre, Genesis, 1999. p.1-8.

MIELNICZUK, J.; BAYER, C.; BESAN, F.M.; LOVATO, T.; FERNÁNDEZ, F.F. \& DEBARBA, L. Manejo de solo e culturas e sua relação com os estoques de carbono e nitrogênio do solo. In: CURI, N.; MARQUES, J.J.; GUILHERME, L.R.G.; LIMA, J.M.; LOPES, A.S. \& ALVAREZ V., V.H., eds. Tópicos em ciência do solo. Viçosa, MG, Sociedade Brasileira de Ciência do Solo, 2003. v.3. p.209-248.

SÁNCHEZ, M.D. Panorama dos sistemas agroflorestais pecuários na América Latina. In: CARVALHO, M.M.; ALVIM, M.J. \& CARNEIRO, J.C., eds. Sistemas agroflorestais pecuários: Opções de sustentabilidade para áreas tropicais e subtropicais. Juiz de Fora, 2001. p.9-17.

SCHROTH, G.; D'ANGELO, S.A.; TEIXEIRA, W.G.; HAAG, D. \& LIEBEREI, R. Conversion of secondary forest into agroforestry and monoculture plantations in Amazônia: Consequences for biomass, litter and soil carbon stocks after 7 years. For. Ecol. Manag., 163:131-150, 2002.

SHARMA, K.L.; MANDAL, U.K.; SRINIVAS, K.; VITTAL, K.P.R.; MANDAL, B.; GRACE, J.K. \& RAMESH, V. Longterm soil management effects on crop yelds and soil quality in a dryland Alfisol. Soil Till. Res., 83:246-259, 2005.

SILVA, I.F. \& MIELNICZUK, J. Ação do sistema radicular de plantas na formação e estabilização de agregados do solo. R. Bras. Ci. Solo, 21:113-117, 1997b.

SILVA, I.F. \& MIELNICZUK, J. Avaliação do estado de agregação do solo afetado pelo uso agrícola. R. Bras. Ci. Solo, 21:313-319, 1997a.
SILVA, J.E. \& RESCK, D.V.S. Matéria orgânica do solo. In: VARGAS, M.A.T. \& HUNGRIA, M., eds. Biologia dos solos dos cerrados. Planaltina, 1997. p.467-524.

SMITH, W.N.; DESJARDINS, R.L. \& GRANT, B. Estimated changes in soil carbon associated with agricultural practices in Canada. Can. J. Soil. Sci., 81:221-227, 2001.

SOHI, S.; MAHIEU, N.; ARAH, J.R.M.; POLWSON, D.S.P.; MADARI, B. \& GAUNT, J.L. A procedure for isolating soil organic matter fractions suitable for modeling. Soil Sci. Soc. Am. J., 65:1121-1128, 2001.

SOLOMON, D.; LEHMANN, J. \& ZECH, W. Land use effects on soil organic matter properties of Chromic Luvisols in semi-arid Northern Tanzania: Carbon, nitrogen, lignin and carbohydrates. Agric. Ecosys. Environ., 78:203-213, 2000.

SOUZA, W.J.O. \& MELO, W.J. Teores de nitrogênio no solo e nas frações da matéria orgânica sob diferentes sistemas de produção de milho. R. Bras. Ci. Solo, 24:885-896, 2000.

STEVENSON, F.J. Humus chemistry: Genesis, composition, reactions. 2.ed. New York, John Wiley \& Sons, 1994. 496p.

STEVENSON, F.J. \& COLE, M.A. Cycles of soil: Carbon, nitrogen, phosphorus, sulfur, micronutrients. 2.ed. New York, John Wiley \& Sons, 1999. 427p.

SWIFT, R.S. Organic matter characterization. In: SPARKS, D.L.; PAGE, A.L.; HELMKE, P.A.; LOEPPERT, R.H.; SOLTANPOUR, P.N.; TABATABAI, M.A.; JOHNSTON, C.T. \& SUMMER, M.E., eds. Methods of soil analysis. Part 3. Chemical methods. 3.ed. Madison, Soil Science Society of America/American Society of Agronomy, 1996. p.1011-1020.

TEDESCO, M.J.; GIANELLO, G.; BISSANI, C.A.; BOHNEN, H. \& VOLKWEIS, S.I. Análise de solo, plantas e outros materiais. 2.ed. Porto alegre, Universidade Federal do Rio Grande do Sul, 1995. 174p.

WRIGHT, A.L.; HONS, F.M. \& MATOCHA JR., J.E. Tillage impacts on microbial biomass and soil carbon and nitrogen dynamics of corn and cotton rotations. Appl. Soil Ecol., 29:85-92, 2005.

XAVIER, F.A.S.; MAIA, S.M.F.; OLIVEIRA, T.S. \& MENDONÇA, E.S. Biomassa microbiana e matéria orgânica leve em solos sob sistemas agrícolas orgânico e convencional na Chapada da Ibiapaba - CE. R. Bras. Ci. Solo, 30:247-258, 2006.

YANG, E.J.; SKOGLEY, E.O.; SCHAFF, B.E. \& KIM, J.J. A simple spectrophotometric determination of nitrate in water, resin and soil extracts. Soil Sci. Soc. Am. J., 62:1108$1115,1998$.

YANG, X.M. \& KAY, B.D. Impacts of tillage practices on total, loose- and oclluded-particulate, and humified organic carbon fractions in soils within a field in Southern Ontario. Can. J. Soil Sci., 81:149-156, 2001. 
ZIBILSKE, L.M.; BRADFORD, J.M. \& SMART, J.R. Conservation tillage induced changes in organic carbon, total nitrogen and available phosphorus in a semi-arid alkaline subtropical soil. Soil Till. Res., 66:153-163, 2002.
ZINN, Y.L.; LAL, R. \& RESCK, D.V.S. Changes in soil organic carbon stocks under agricultural in Brazil. Soil Till. Res., 84:28-40, 2005. 